\title{
Dual Mode Ferroelectric Transistor based Non-Volatile Flip-Flops for Intermittently-Powered Systems
}

\author{
S. K. Thirumala ${ }^{1}$, A. Raha*, ${ }^{2}$, H. Jayakumar ${ }^{*}{ }^{3}$, K. Ma $^{4}$, V. Narayanan ${ }^{4}$, V. Raghunathan ${ }^{1}$ and S. K. Gupta ${ }^{1,4}$ \\ Purdue University ${ }^{1}$, Intel Corp. ${ }^{2}$, Qualcomm Inc. ${ }^{3}$, Pennsylvania State University ${ }^{4}$. Email: sthirum@purdue.edu
}

\begin{abstract}
In this work, we propose dual mode ferroelectric transistors (D-FEFETs) that exhibit dynamic tuning of operation between volatile and non-volatile modes with the help of a control signal. We utilize the unique features of D-FEFET to design two variants of non-volatile flip-flops (NVFFs). In both designs, D-FEFETs are operated in the volatile mode for normal operations and in the non-volatile mode to backup the state of the flip-flop during a power outage. The first design comprises of a truly embedded non-volatile element (D-FEFET) which enables a fully automatic backup operation. In the second design, we introduce need-based backup, which lowers energy during normal operation at the cost of area with respect to the first design. Compared to a previously proposed FEFET based NVFF, the first design achieves 19\% area reduction along with 96\% lower backup energy and 9\% lower restore energy, but at $14 \%-35 \%$ larger operation energy. The second design shows $11 \%$ lower area, $21 \%$ lower backup energy, $16 \%$ decrease in backup delay and similar operation energy but with a penalty of $17 \%$ and $19 \%$ in the restore energy and delay, respectively. System-level analysis of the proposed NVFFs in context of a state-of-the-art intermittently-powered system using real benchmarks yielded 5\%-33\% energy savings.
\end{abstract}

\section{CCS Concepts}

Hardware $\rightarrow$ Emerging Technologies $\rightarrow$ Analysis and design of emerging devices and systems.

\section{Keywords}

Check-pointing; FEFETs; Hysteresis; Non-volatile flip-flop.

\section{INTRODUCTION}

Energy harvesting from ambient sources has been extensively studied as a promising candidate to enable energy autonomous systems. In the near future, it is predicted that a number of systems will be powered using harvested energy including, toxic gas sensors, portable gadgets and medical implants [1-ㄹ] However, scavenged energy from ambient sources such as solar, thermal and vibrations exhibits an erratic nature with intermittent power supply $\left(V_{D D}\right)$. Such power failures have a drastic impact on standard CMOS logic, suffering from inefficient reboots and

Permission to make digital or hard copies of all or part of this work for personal or classroom use is granted without fee provided that copies are not made or distributed for profit or commercial advantage and that copies bear this notice and the full citation on the first page. Copyrights for components of this work owned by others than ACM must be honored. Abstracting with credit is permitted. To copy otherwise, or republish, to post on servers or to redistribute to lists, requires prior specific permission and/or a fee. Request permissions from Permissions@acm.org.

ISLPED '18, July 23-25, 2018, Seattle, WA, USA

(C) 2018 Association for Computing Machinery.

ACM ISBN 978-1-4503-5704-3/18/07...\$15.00

https://doi.org/10.1145/3218603.3218653

${ }^{*}$ Contributed to the research while affiliated with Purdue University. rollbacks [3]. Therefore, it becomes important to back up the state of a logic system to alleviate the loss in computation progress.

Non-volatile computing is an emerging solution to mitigate computation progress loss due to unexpected power failures [3] . Systematic consistency-aware check-pointing mechanisms have been proposed to avoid data inconsistency and computation errors due to power failures [4]. This is achieved by backing up the states of a circuit such as, on-chip memory, flip-flops (FFs) and registers into an off-chip non-volatile memory (NVM). However, this incurs significant energy/delay overheads due to long distance data transmissions and constrained parallelism. Embedded non-volatile computing is an attractive alternate method to backup the computation states into a local on-chip NVM, eliminating the transmission overheads. Several NVFF designs using memristors [ㄷ], magnetic tunnel junctions []ㅡ and resistive RAMs [7] as local non-volatile elements have been proposed with on-demand backup/restore (B/R). However, they may incur area overheads due to the incorporation of a $B / R$ module [6]. Also, high write current during backup increases their power consumption [7]. Ferroelectric (FE) capacitor based designs have also been proposed, utilizing their property of polarization $(P)$ retention in the absence of electric field $(E)$ [8]. However, low distinguishability between their non-volatile states degrades the robustness during restore operation.

The recent advent of ferroelectric transistors (FEFETs) with the possibility to integrate FE in the gate stack of a transistor has led to a new era for logic-memory synergy [9]. Lately, FEFET based NVFFs have been proposed with a potential to overcome the challenges in FE capacitor based designs [10-11]. The innovation stems from utilizing the three-terminal non-volatile transistor to improve distinguishability and simplify the restore operation. However, these designs also contain a $\mathrm{B} / \mathrm{R}$ module driven by control signals, leading to higher switching energy/delay. Hence, there is a need to optimize the $\mathrm{B} / \mathrm{R}$ module by exploring new device technologies that leverage the opportunities offered by FE.

To that effect, we propose a dual mode FEFET (D-FEFET) which exhibits a unique characteristic of dynamic tuning between volatile and non-volatile modes. Exploiting such distinct features, we propose 2 variants of energy-efficient NVFF designs (referred to as DNVFF-1 and DNVFF-2). The contributions of this paper are:

- We introduce D-FEFET and extensively explain its characteristics including run-time modulation of hysteresis.

- We utilize D-FEFET in the design of NVFF with fully automatic backup without any B/R module (DNVFF-1), demonstrating significantly lower area and backup/restore energy compared to previous FEFET based NVFF [10].

- We propose another NVFF (DNVFF-2) with need-based backup enabled by compact low power B/R module, showing lower area and backup energy/speed compared to [10].

- We carry out a system-level analysis of the proposed NVFFs for intermittently-powered systems. 


\section{MODELING AND CALIBRATION}

To evaluate the proposed designs, we employ a SPICE model for FEFETs based on time-dependent Landau Khalatnikov (LK) equations coupled with predictive technology models for the transistor [12]. We perform our analysis for $10 \mathrm{~nm}$ FinFETs showing the applications of the proposed techniques in scaled technologies. However, considering the fact that some applications may prefer older technologies to optimize leakage, we also analyze our NVFFs in 130nm technology for system-level analysis (as discussed later). For CMOS process compatibility, we use experimental data of $\mathrm{Hf}_{0.7} \mathrm{Zr}_{0.3} \mathrm{O}_{2}$ with remnant polarization $\left(P_{R}\right)=20 \mu \mathrm{C} / \mathrm{cm}^{2}$ and coercive field $\left(E_{C}\right)=1 \mathrm{MV} / \mathrm{cm}[\underline{13}]$, to calibrate our model (Fig. 1(a)). The static coefficients $(\alpha, \beta$ and $\gamma)$ of the LK equation were extracted from the polarization versus voltage $(P-$ $V)$ loop measured from experiment [13] (Fig. 1(b)). The kinetic coefficient $(\rho)$ was calculated for $P$ switching time of $\sim 100$ ps [14] .

\section{DUAL MODE FEFET (D-FEFET)}

\subsection{FEFET Background}

FEFETs are emerging devices $[\underline{9}, \underline{12}]$, in which FE is integrated in the gate stack of a transistor above the dielectric (DE). An optional metal may also be used in between FE and DE layers. The capacitance of FE couples with that of the underlying FET leading to unique characteristics: (i) sub $60 \mathrm{mV} /$ decade sub-threshold swing for low power logic [12] and (ii) non-volatile transistor operation (due to $P$ retention in the absence of $E$ ) [9]. It has been shown that, by changing the capacitance ratio between the FE and the underlying FET, for instance by varying the FE thickness $\left(T_{F E}\right)$, one can operate the FEFETs in the non-volatile (memory) or volatile (logic) mode [15]. Although, static tuning of $T_{F E}$ enables design-time optimization of FEFETs, run-time tuning between memory and logic modes can open new avenues for circuit design. In this work, we propose D-FEFET, which achieves dynamic reconfigurability between the volatile and non-volatile modes.

\subsection{Dual Mode FEFET Design}

Fig. 2(a) shows the device structure of D-FEFET. It consists of two gate stacks namely GATE $\left(\mathrm{FE}_{\mathrm{G}}\right)$ and CONTROL ( $\left.\mathrm{FE}_{\mathrm{C}}\right)$. The internal metal (IM) gate in the two stacks is common and serves as the gate of the underlying FinFET. This structure can be realized with minimal changes to the FEFET fabrication process. First, the standard process flow is used to obtain a FinFET with one/two of the fins in the middle etched and the gate stack formed up till the IM. This is followed by FE and gate metal deposition, and a selective etch (enabled by the spacing between two sets of fins) to isolate the two FE stacks. Both the FE stacks have the same $T_{F E}$. The ratio of capacitance of the $\mathrm{FE}_{\mathrm{G}}$ and $\mathrm{FE}_{\mathrm{C}}$ stack is $C_{F E G}$ : $C_{F E C}$ $=2: 1$. This is because $\mathrm{FE}_{\mathrm{G}}$ is formed on two fins while $\mathrm{FE}_{\mathrm{C}}$ is formed on a single fin. Note, D-FEFETs can also be realized using
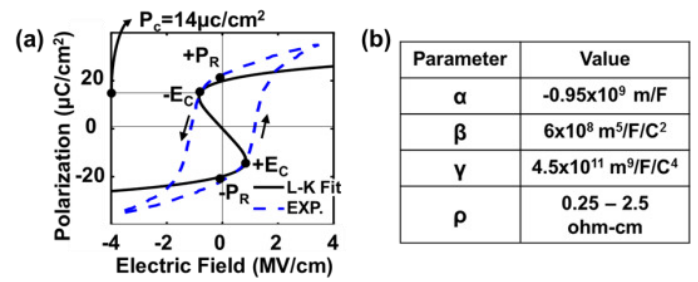

Figure 1. (a) Calibration of model with experimental $P_{R}$ and $E_{C}$ values [13] (b) Extracted FE parameters for the LK model

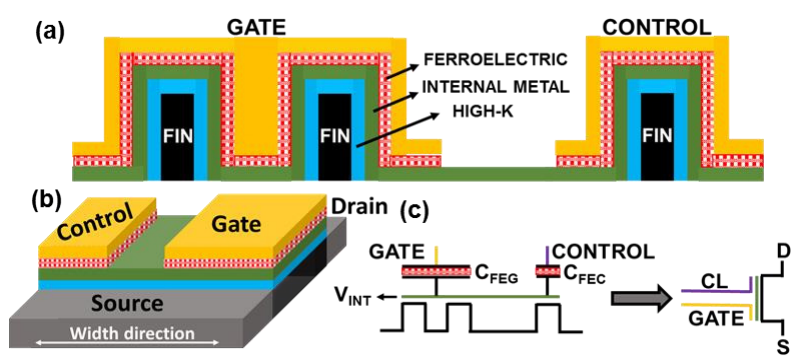

Figure 2. (a) Proposed D-FEFET with 3 fins (b) D-FEFET realized using planar FET (c) Schematic representation

planar technologies with similar process flow (Fig. 2(b)) leveraging contact over active gate process [16]. Fig. 2(c) shows the equivalent schematic of D-FEFET with the connection of $C_{F E G}$ and $C F E C$ with the underlying FET. Note, our simulations consider the parasitic capacitances between the FE stacks. The role of FEC is to modulate the modes of operation as explained next.

\subsection{Dual Mode FEFET Operation}

The control terminal of the D-FEFET interacts with $\mathrm{FE}_{\mathrm{G}}$ and the underlying transistor to enable reconfigurability. The internal metal potential $\left(V_{I N T}\right)$ is designed to be affected to a larger extent by the polarization $(P)$ of the regular gate stack $\left(P_{F E G}\right)$ compared to control stack $\left(P_{F E C}\right)$ by ensuring $C_{F E G}>C_{F E C}$. Note that because of the presence of the common IM, $P_{F E G}$ and $P_{F E C}$ interact with each other, which yields unique characteristics, as discussed below.

(i) Non-volatile ('NV') mode: For the ' $N V$ ' mode, we apply control voltage $\left(V_{C L}\right)=0$. Hence, voltage across FEC $\left(V_{F E C}\right)=-V_{I N T}$ and voltage across $\mathrm{FE}_{\mathrm{G}}\left(V_{F E G}\right)=V_{G S}-V_{I N T}$ (where $V_{G S}$ is the gate voltage). Note, since $C_{F E G}>C_{F E C}, V_{I N T}$ is affected to a larger extent by capacitive coupling of $F E_{G}$ than $F E_{C}$. The coercive voltages $\left(V_{C}\right)$ of the FE are designed (with $T_{F E}<11 \mathrm{~nm}$ ) such that $P$ switching occurs in both the gate stacks, as described below. Let us consider $V_{G S}$ sweep from a negative to positive value and back (with drain voltage $\left.V_{D S}=0 \mathrm{~V}\right)$. To start with, application of negative $V_{G S}(=-$ $0.8 V$ ) yields negative $P_{F E G}(-P)$ and negative $V_{I N T}$ (Fig. 3(a, c)). In turn, negative $V_{I N T}$ yields a positive $V_{F E C}$ (since $V_{F E C}=-V_{I N T}$ ), leading to positive $P_{F E C}$ (Fig. 3(b)). As $V_{G S}$ is increased, $P_{F E G}$ switches to a positive polarization $(+P)$ when $V_{F E G}$ exceeds $V_{C}$. $P_{F E G}$ switching results in an increase in $V_{I N T}$ to a positive value (Fig. 3(c)) due to the capacitive coupling of $C_{F E G}$. Hence, $V_{F E C}\left(=-V_{I N T}\right)$ becomes negative which yields negative $P_{F E C}$ (Fig. 3(b)). Similarly, during the reverse sweep of $V_{G S}, P_{F E G}$ switches from $+P$ to $-P$, yielding $V_{I N T}<0, V_{F E C}>0$ and $P_{F E C}>0(+P)$. Thus, in the ' $N V$ ' mode $\left(V_{C L}=0\right), \mathrm{FE}_{\mathrm{G}}$ and $\mathrm{FE}_{\mathrm{C}}$ always exhibit opposite polarizations. Due to this opposing effect of $\mathrm{FE}_{\mathrm{C}}$, the effect of $\mathrm{FE}_{\mathrm{G}}$ on $V_{I N T}$ is reduced compared to that in a standard FEFET. Hence, lower $\left|V_{I N T}\right|$ is obtained in D-FEFETs (Fig. 3(c)). This increases the critical $\left|V_{G S}\right|$ for $P_{F E G}$ switching (since $V_{C}$ of $\left.\mathrm{FE}_{G}=\left|V_{G S}\right|-\left|V_{I N T}\right|\right)$. As a result, a larger hysteresis is observed in D-FEFETs compared to standard

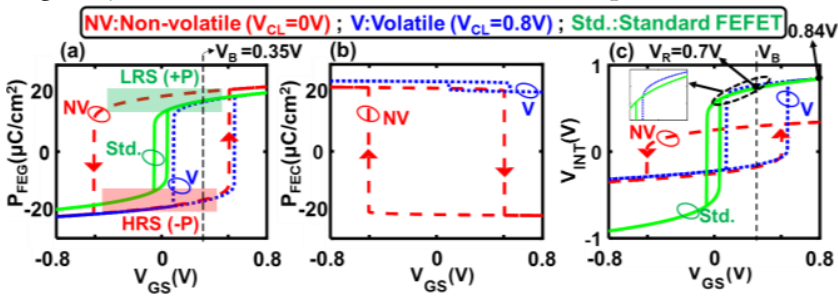

Figure 3. Polarization of (a) Gate stack, (b) Control stack and (c) Internal metal voltage versus gate voltage. $T_{F E}=8 \mathrm{~nm} ; V_{D S}=0 \mathrm{~V}$ 
FEFETs with two fins (Fig. 3(a)). Note, $P_{F E G}$ (with bi-stability at $V_{G S}=0$ in the ' $N V$ ' mode) define the low and high resistance states (LRS/ $+P$ and HRS/ $-P$ ) of the D-FEFET since it influences the channel electrostatics to a larger extent than $P_{F E C}$.

ii) Volatile (' $V$ ') mode: To operate D-FEFETs in the ' $V$ ' mode, we apply $V_{C L}=V_{D D}(0.8 \mathrm{~V})$. Due to positive $V_{C L}, P_{F E C}$ remains at $+P$ for the entire range of $V_{G S}\left(V_{F E C}>+V_{C}\right.$ always; Fig. 3(b)). For $V_{G S}<$ $0(-0.8 V), P_{F E G}=-P$ is obtained. This yields negative $V_{I N T}$ (despite positive $P_{F E C}$ because $\left.C_{F E G}>C_{F E C}\right)$. As shown in Fig. 3(c), $V_{I N T}$ is slightly less negative than the ' $N V$ ' mode $\left(V_{C L}=0 V\right)$ due to the effect of the positive $P_{F E C}$ (since $V_{C L}=V_{D D}$ ). This results in a slightly larger $V_{G S}$ required for $P_{F E G}$ switching from $-P$ to $+P$ (since $V_{C}$ of $\mathrm{FE}_{\mathrm{G}}=V_{G S}-V_{I N T}$ ). During the reverse $V_{G S}$ sweep (from positive to negative values), both $P_{F E G}$ and $P_{F E C}$ are $+P$ initially. Hence, $V_{I N T}$ is much larger compared to ' $N V$ ' mode. In fact, $V_{I N T}$ is even larger than FEFETs (inset; Fig. 3(c)) due to $V_{C L}=V_{D D}\left(P_{F E C}=+P\right)$. A large $V_{I N T}$ enables $\mathrm{FE}_{\mathrm{G}}$ switching from $+P$ to $-P$ at $V_{G S}>0\left(V_{G S}-V_{I N T}=-\right.$ $V_{C}$ ), resulting in the ' $V$ ' mode (one stable state at $V_{G S}=0$, Fig. 3(a)).

The application of $V_{D S}$ decreases $P[17]$ and increases VINT, due to the capacitive coupling of drain and IM. This results in shifting of the $P-V$ characteristics towards the right. Fig. 4(a) shows the drain current vs $V_{G S}$ characteristics. Note that for logic design, it is critical to optimize $T_{F E}$ to ensure switching of $P_{F E G}$ from $-P$ to $+P$ occurs within the 0 to $V_{D D}$ window.

The ON current $(I O N)$ of D-FEFET (with $T_{F E}=8 \mathrm{~nm}$ ) in the ' $V$ ' mode shows about 36\% increase compared to FinFET (Fig. 4(a)). This is because $\mathrm{FE}_{\mathrm{G}}$ operates in the negative $P-V$ region [12] in the ON state $\left(V_{F E G}<0, P>0\right)$, yielding a voltage step-up action [12] $\left(V_{I N T}\right.$ $>V_{G S}$ since $V_{I N T}=V_{G S}-V_{F E G}=0.84 \mathrm{~V}$; Fig. $\left.3(\mathrm{c})\right)$. In the ' $N V^{\prime}$ ' mode, the currents at $V_{G S}=0 V$ (defining HRS and LRS), exhibit a large ratio (> $10^{5}$; Fig. $4(\mathrm{~b})$ ), yielding excellent distinguishability.

By utilizing the dynamic reconfigurability between ' $V$ ' and ' $N V$ ' modes of D-FEFETs, we propose two NVFF designs next.

\section{NVFF with AUTO BACKUP: DNVFF-1}

\subsection{Circuit Design}

Our first design (DNVFF-1) features a fully automatic backup operation by virtue of a truly embedded NV element (D-FEFET) in the FF (Fig. 5(a)). This is unlike previous designs [ $\underline{5}-\underline{8}]$ where the $\mathrm{NV}$ element is in a separate $\mathrm{B} / \mathrm{R}$ module, interfacing the $\mathrm{FF}$ via external control signals. (Note, embedding of FEFET in FF is challenging because of the requirement of $V_{G S}<0$ for $P$ switching). The slave latch is re-designed utilizing two D-FEFETs. The rest of the other devices are standard transistors. One of the D-FEFET is used in the pull-down network (M4) of the feedback path while the other as nMOS (M6) of inverter INV1. The control gates of DFEFETs are tied together, which can dynamically reconfigure their operation between the ' $V$ ' and ' $N V$ ' modes as discussed before. The D-FEFETs are used in the ' $V$ ' mode for the normal and restore operations, while in the ' $N V$ ' mode during backup operation. The
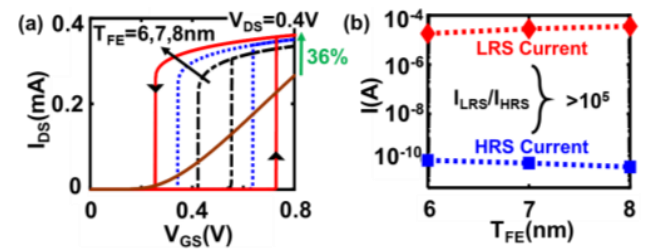

Figure 4. (a) Transfer characteristics of the D-FEFET and 3-fin FinFET. (b) LRS-HRS currents for various $T_{F E}$ in the ' $N V$ ' mode.

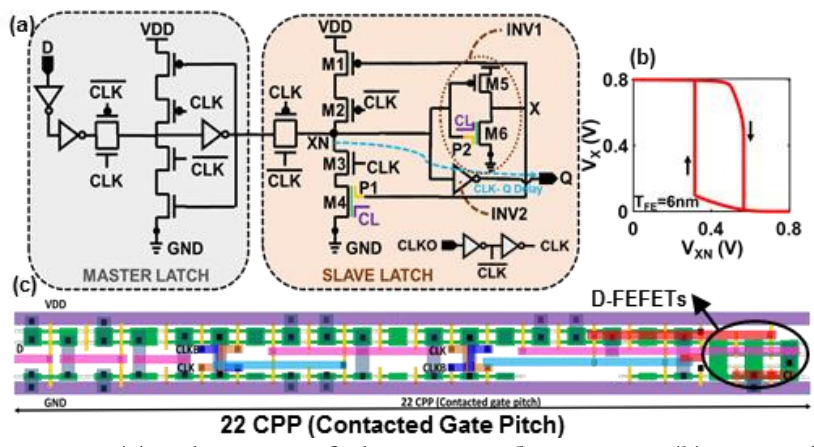

Figure 5. (a) Schematic of the proposed DNVFF-1 (b) VTC of INV1 (c) Layout of DNVFF-1. \#fins for std. $n M O S=1 ; p M O S=2$ embedding of D-FEFET affects the Clock-to-Q (C-to-Q) delay and energy consumption of the FF during normal operation since, $P$ switching may not be as fast as standard FETs. To alleviate the effect on C-to-Q, inverter INV2 is employed to bypass INV1 and obtain output Q. Standard FETs are sized considering optimized area and similar C-to-Q delay for rising/falling outputs. All simulations are carried out with FO4 load at $\mathrm{Q}$ with input slew rate $=100 \mathrm{ps}$ at $V_{D D}=0.8 \mathrm{~V}$ (Transient waveforms in Fig. 6).

\subsection{Circuit Operation}

(i) Normal Operation: During the normal operation, $V_{C L}$ of the D-FEFETs is driven to $V_{D D}$ to operate them in the ' $V$ ' mode, in order to function as a switch $\left(\mathrm{OFF} / \mathrm{ON}\right.$ when $\left.V_{G S}=0 / V_{D D}\right)$. Thus, the normal operation of DNVFF-1 is similar to the conventional FF (Fig. 6(b)). Note, the voltage transfer characteristics (VTC) of INV1 shows hysteretic behavior (Fig. 5(b)), exhibiting improved hold stability. $P_{F E G}$ of D-FEFETs switches as per its $V_{G S}$. Thus, when $\mathrm{Q}$ is at $V_{D D}, \mathrm{X}$ and $\mathrm{XN}$ are at $V_{D D}$ and 0 respectively and $\mathrm{FE}_{\mathrm{G}}$ of M4 and M6 store $+P$ and $-P$ respectively (Fig. 7(a)). Similarly, when $Q$ is at $0, \mathrm{FE}_{\mathrm{G}}$ of $\mathrm{M} 4$ and $\mathrm{M} 6$ store $-P$ and $+P$, respectively. Note, using INV2 to bypass INV1 in the C-to-Q path has minimal impact on the delay. However, the energy consumption increases due to $P$ switching. These aspects are discussed later.

(ii) Auto Backup Operation: One of the most appealing features of DNVFF-1 is its automatic backup operation, without the use of external circuitry or signals. This makes it very useful for systems requiring frequent check-pointing. PFEG of D-FEFETs correspond to the storage nodes $\mathrm{X}$ and $\mathrm{XN}$, as discussed before. During power

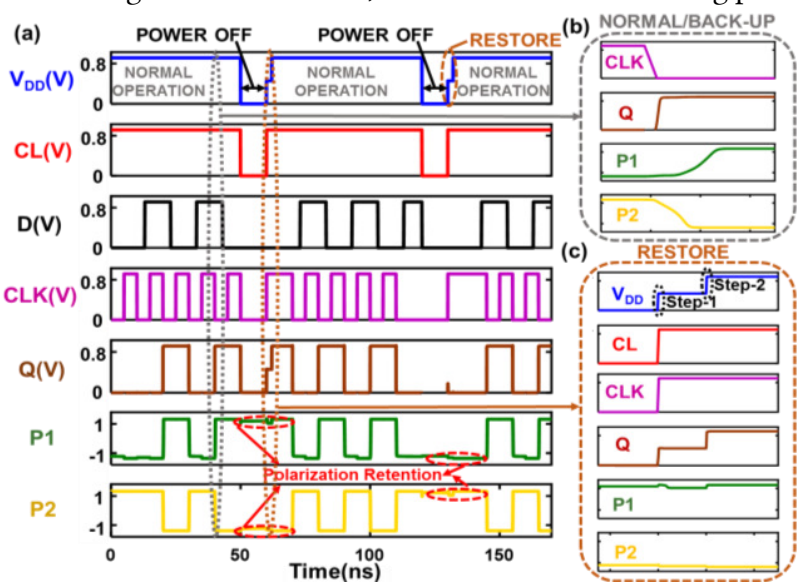

Figure 6. (a) Transient waveforms of DNVFF-1. (b) Normal and Auto-backup operation showing polarization switching (c) 2 step restore operation. The polarizations are normalized with $P_{\mathrm{C}}=14 \mu \mathrm{C} / \mathrm{cm}^{2}$ (see Fig. 1(a)). $T_{\mathrm{FE}}=6 \mathrm{~nm}$ 


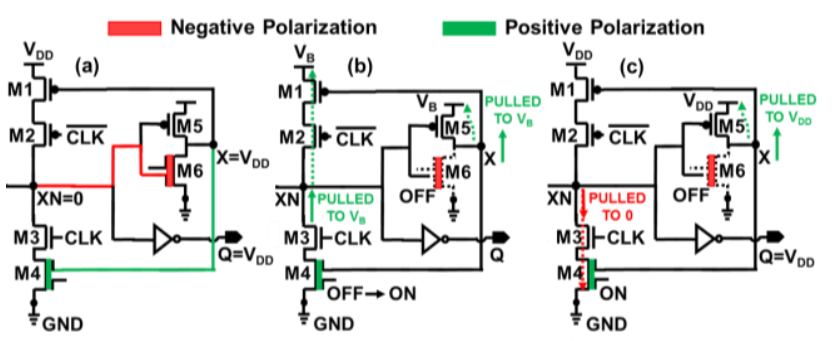

Figure 7. Slave latch schematic showing (a) Normal Operation (b) Step-1 and (c) Step-2 of restore operation of the state $Q=1$

outage, $V_{C L}$ becomes 0 and the D-FEFETs dynamically and automatically change operation from the ' $V$ ' to ' $N V$ ' mode. This leads to retention of $P_{F E G}$ in M4/M6 corresponding to $\mathrm{X} / \mathrm{XN}$. Note, $\mathrm{FE}_{\mathrm{C}}$ of only one of the D-FEFETs undergoes $+P$ to $-P$ switching when power shuts down (to maintain opposite $P$ in $\mathrm{FEg}_{\mathrm{G}}$ and FEc in ' $N V$ ' mode; Fig. 3(a, b)). This results in minimal backup energy.

(iii) Restore Operation: Like backup, the restore operation does not require any additional circuitry. Note that the IM gate potential, which stores the information of the resistance state of the D-FEFETs (corresponding to $\mathrm{X}$ and $\mathrm{XN}$ ) during the ' $N V$ ' mode, is floating. Therefore, over time, gate leakage can lead to discharging of $V_{I N T}$ to $O V$ (HRS). However, it is important to note that $P_{F E G}$, containing the information of the storage nodes of $\mathrm{FF}$, is still retained [18]. In our analysis, we have considered the worstcase scenario, where $V_{I N T}$ discharges to $O V$ during the ' $N V$ ' mode, and propose a two-step restore process. (a) Step-1: $C L K$ and $V_{C L}$ are asserted when power supply is restored. $V_{D D}$ of the FF is raised till $V_{B}(0.35 \mathrm{~V})$ to bias the gates of the D-FEFETs (X and XN) at an intermediate voltage within the hysteresis of the ' $V$ ' mode (see Fig. 7(b); Fig. 3(a)). This ensures that $P_{F E G}$ of the two D-FEFETs are undisturbed when $V_{C L}=V_{D D}$. However, this step leads to $P$ switching in $\mathrm{FE}_{\mathrm{C}}$ of the D-FEFET whose initial $P_{F E C}$ was $-P$ (because in the ' $V$ ' mode $P_{F E C}=+P$ always; Fig. 3(b)). This switching of $P_{F E C}$ to $+P$ leads to charging of $V_{I N T}$ to $V_{R}(0.7 \mathrm{~V})$ in the D-FEFET (Fig. 8(a); Fig. 3(c)). Thus, the D-FEFET with initial $P_{F E C}=-P$ $\left(P_{F E G}=+P\right)$ undergoes $V_{I N T}$ restoration (LRS). The D-FEFET storing $+P$ in its $F_{C}$ does not undergo any switching and $V_{I N T}$ remains at OV (HRS; Fig. 8(b)). (b) Step-2: After $V_{I N T}$ restoration in Step-1, $V_{D D}$ is raised to $0.8 \mathrm{~V}$ (full $V_{D D}$ ) and FF state is restored depending on the resistance states of the D-FEFETs. Note, to enhance the robustness to variations, the ' $V$ ' mode hysteresis width can be increased by optimizing $T_{F E}$ (Fig. 4(a)). Also note, restore biasing using slow $V D D$ ramp-up has also been employed in [19].

Let us explain the restore operation further considering that $\mathrm{Q}$ was at $V_{D D}$ before the power outage. As discussed before, $\mathrm{FE}_{\mathrm{G}}$ of $\mathrm{M} 4$ and M6 store $+P$ and $-P$ respectively during auto-backup. Step1 includes the assertion of $C L K, V_{C L}$ (' $V$ ' mode) and powering $V_{D D}$ to $V_{B}$. M1 and M5 turn ON as $V_{D D}$ is powered up (Fig. 7(b)). This switches $P_{F E C}$ of M4 from $-P$ to $+P$ leading to $V_{I N T}$ restoration (to $V_{R}=0.7 V$; LRS). $V_{I N T}$ of M6 remains at $0 V$ (HRS). During Step-2 $\left(V_{D D}\right.$ raised till $0.8 \mathrm{~V}$ ), stronger pull-down by virtue of LRS of M4 and M3 keeps the node $\mathrm{XN}$ at ' 0 ' overcoming the effect of M1, while M5 pulls $\mathrm{X}$ to $V_{D D}$. The large LRS-HRS ratio in D-FEFETs along with the cross-coupled action of the slave latch, leads to restoration of

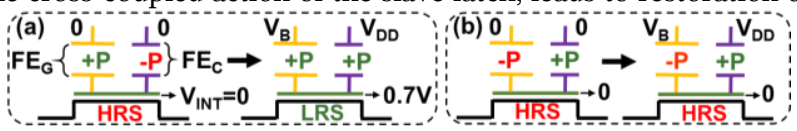

Figure 8. Step-1 of restore operation for $\mathrm{V}_{\mathrm{INT}}$ restoration when (a) initial $P_{\mathrm{FEC}}=-\mathrm{P}$ and $(\mathrm{b}) \mathrm{P}_{\mathrm{FEC}}=+\mathrm{P} . \mathrm{V}_{\mathrm{B}}=0.35 \mathrm{~V} ; \mathrm{V}_{\mathrm{DD}}=0.8 \mathrm{~V}$ the state $\mathrm{X}=V_{D D}$ and $\mathrm{XN}=0$ as illustrated in Fig. 7 (c). The transient waveforms of the restore operation are shown in Fig. 6(c).

\section{NVFF with GATED BACKUP: DNVFF-2}

\subsection{Circuit Design}

We propose another NVFF design, which features need-based backup, and therefore requires an external $\mathrm{B} / \mathrm{R}$ module to trigger the B/R operations (Fig. 9(a)). Since, backup occurs only when the power is about to go OFF, DNVFF-2 mitigates the large operational energy $\left(E_{O P}\right)$ cost incurred in DNVFF-1. DNVFF-2 employs a $\mathrm{B} / \mathrm{R}$ module with 5 transistors including $2 \mathrm{D}$-FEFETs unlike [10] which uses 8 . Transistors $\mathrm{M} 1$ and $\mathrm{M} 2$ connect the $\mathrm{B} / \mathrm{R}$ module to the slave latch. M4 and M5 are the D-FEFETs which store the states of the FF. Transistor M3 connects the drains of M4 and M5 to ensure PFEG of $\mathrm{M} 4 / \mathrm{M} 5=-P$ during the normal operation as explained next.

\subsection{Circuit Operation}

(i) Normal Operation: During the normal operation (transient waveforms in Fig. 9(b)), M1 and M2 are turned OFF isolating the $\mathrm{FF}$ from the $\mathrm{B} / \mathrm{R}$ module. Thus, $\mathrm{D}$-FEFETs have minimal impact on the normal operation of the FF. In the $\mathrm{B} / \mathrm{R}$ module, M3 is turned $\mathrm{ON}$ and $V_{C L}$ is asserted to operate D-FEFETs M4 and M5 in ' $V$ ' mode. Just before the normal operation (i.e. after the restore operation finishes), one of the D-FEFETs is in LRS. By turning ON M3 we ensure that the drains/gates of both the D-FEFETs are driven to $0 V$. With $V_{G S}$ of D-FEFETs $=0$ and $V_{C L}=V_{D D}, F E_{G}$ of M4 and M5 store $-P$ (HRS) (Fig.3 (a)). Operating D-FEFETs in HRS is critical during the initial stages of backup, as discussed next.

(ii) Backup Operation: For backup, M1 and M2 are turned ON with M3 OFF and $V_{C L}=0$ ('NV' mode). At the beginning of backup, both the D-FEFETs are in the HRS (as discussed above). Now, depending on the voltages of nodes $\mathrm{X}$ and $\mathrm{XN}$, one of the DFEFETs receives $V_{G S}=0$ and the other receives $V_{G S}=V_{D D}$. For

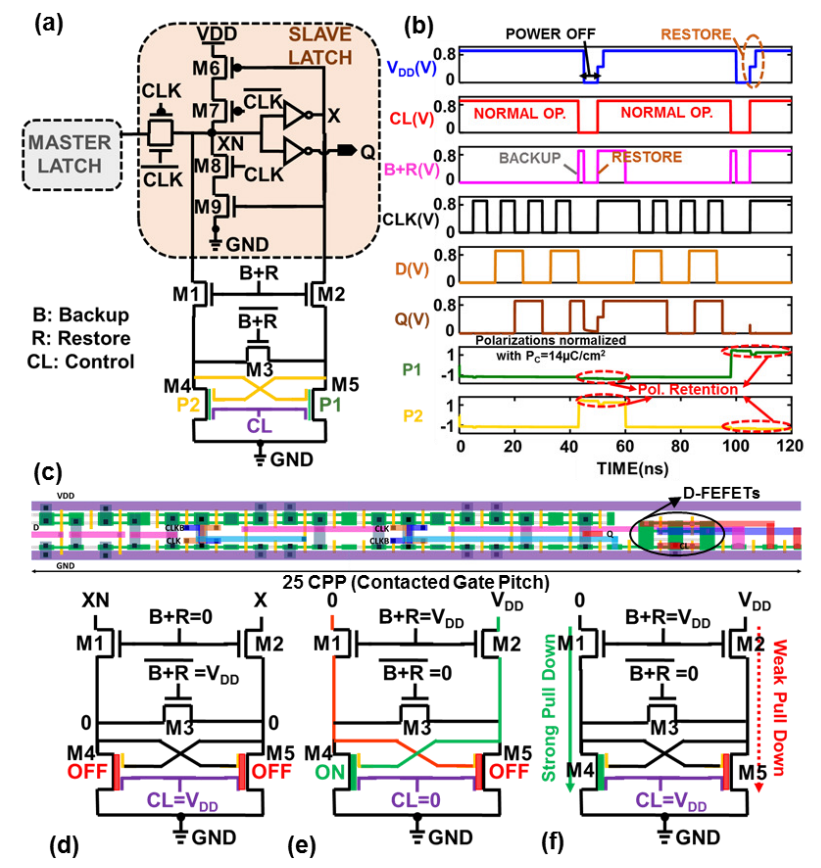

Figure 9. (a) Schematic of the proposed DNVFF-2 (b) Transient waveforms showing normal, backup and restore operations. (c) Layout of DNVFF-2. (d) Normal (e) Backup and (f) Restore (step2) operations in the $B / R$ module of DNVFF-2. $T_{F E}=6 \mathrm{~nm}$ 

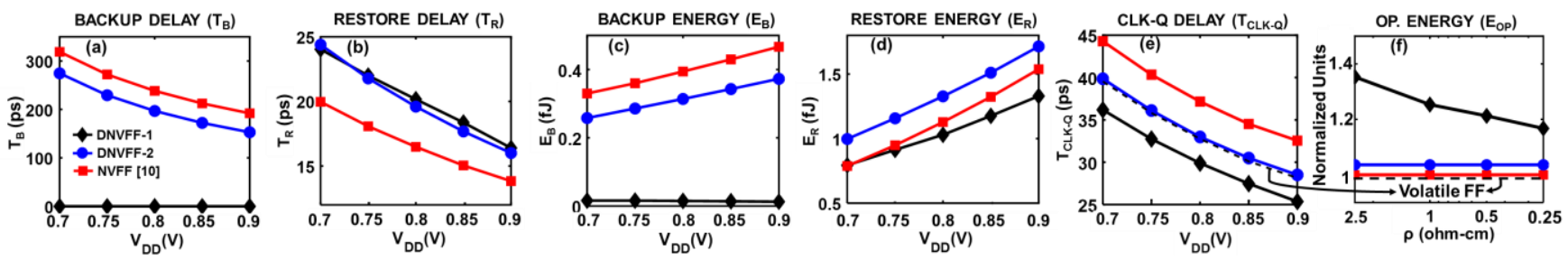

Figure 10. (a) Backup Delay (b) Restore Delay (c) Backup Energy (d) Restore Energy and (e) Clock-to-Q delay versus VDD. (f) Normalized Operation Energy (w.r.t standard volatile FF) vs Kinetic coefficient ( $\rho$ ) for DNVFF-1, DNVFF-2 and previously proposed NVFF [10]. Note, we optimize $T_{F E}$ in FEFET $(11 \mathrm{~nm})$ and D-FEFET $(6 \mathrm{~nm})$ to equalize the hysteresis width.

example, consider the case where $\mathrm{X}=V_{D D}$ and $\mathrm{XN}=0$, which brings $V_{G S}$ of M4 to $V_{D D}$ and M5 to 0 . This results in M4 switching its state to LRS $(+P)$; while M5 remains in HRS (-P) (Fig. 9(e)). In this manner, the state of the FF is stored as $P_{F E G}$ of D-FEFETs.

To understand why D-FEFETs must be in HRS before backup, let us consider a case when this condition is not met. Let $\mathrm{Q}=0$ (i.e., $\mathrm{XN}=V_{D D}$ and $\mathrm{X}=0$ ) before backup. If $\mathrm{M} 4$ and M5 store LRS $(+P)$ and HRS $(-P)$ respectively at the onset of backup, then this would result in $\mathrm{M} 1$ and M4 pulling down $\mathrm{XN}$ to a value $<V_{D D}$ (determined by the resistance ratio of M6-M7 and M1-M4). This voltage at XN might not be enough for $-P$ to $+P$ switching in M5, leading to incorrect backup. Therefore, by design, we ensure M4 and M5 are in HRS at the beginning of backup, as described in Section 5.2.i.

(iii) Restore Operation: When power supply is restored, M1 and M2 are turned ON. The restore operation employs the 2-step process similar to DNVFF-1 (see Section 4.2.iii) and exploits large distinguishability in D-FEFETs along with the cross-coupled action of the slave latch to restore the states of the FF (Fig. 9(f)).

\section{CIRCUIT ANALYSIS AND RESULTS}

In this section, we analyze the proposed DNVFFs and compare them to the NVFF in [10]. Since the design in [11] employs a different mechanism for $\mathrm{B} / \mathrm{R}$ (independent of FF topology), we focus on comparison with [10] (which uses FEFETs in conjunction with cross-coupled inverter action similar to our DNVFFs).

(i) Layout Analysis: A significant advantage of DNVFF-1 is that the $\mathrm{B} / \mathrm{R}$ is achieved without any external module. DNVFF-1 (Fig. 5(c)) shows $19 \%$ lower area with respect to (w.r.t) [10]. DNVFF-2 employs a $\mathrm{B} / \mathrm{R}$ module but uses lower number of transistors w.r.t [10] yielding 11\% lower area (Fig. 9(a, c)). DNVFF-1 and DNVFF-2 show $16 \%$ and $31 \%$ increase in area respectively, w.r.t volatile FF. All layouts are drawn considering scalable CMOS rules [20].

(ii) Backup Delay $\left(T_{B}\right)$ and Energy $\left(E_{B}\right)$ : Fig. 10(a, c) shows the $T_{B}$ and $E_{B}$ of DNVFF-1, DNVFF-2 compared to [10]. Due to automatic backup in DNVFF-1, $T_{B} \sim 0$ ps. Switching of FEc during power outage (as explained before) consumes a very little amount of energy leading to $96 \%$ lower $E_{B}$ w.r.t [10]. This is because: (i) only one of the FEC of D-FEFETs undergoes $P$ switching, (ii) lower capacitance of $\mathrm{FE}_{\mathrm{C}}$ compared to $\mathrm{FE}_{\mathrm{G}}$ and (iii) no external signal switching. DNVFF-2 also has an average of $16 \%$ and $21 \%$ lower $T_{B}$ and $E_{B}$ respectively, over a range of $V_{D D}$. This is attributed to only one FE switching unlike two in [10] (Section 5.2.ii).

(iii) Restore Delay $\left(T_{R}\right)$ and Energy $\left(E_{R}\right)$ : The energy and delay comparison in the restore operation is shown in Fig. 10(b, d). The average decrease in $E_{R}$ over a range of $V_{D D}$ for DNVFF-1 is around $9 \%$. This is because of the lower number of switching control signals required in DNVFF-1 due to the absence of an external $\mathrm{B} / \mathrm{R}$ module. $E_{R}$ of DNVFF-2 is $17 \%$ higher because of $P$ switching in the FEc, which is absent in [10]. $T_{R}$ of DNVFF-1 and DNVFF-2 increases by $19 \%$, which is attributed to the two-step restore operation. Higher $T_{R}$ incurred might not be much of a concern for intermittently-powered systems, which operates at $\sim \mathrm{MHz}$

(iv) Clock-to- $Q$ delay (TCLK-Q) and Operation Energy (EOP): $T_{C L K-Q}$ of DNVFF-1 is $20 \%$ lower w.r.t [10], because of the higher capacitance at $\mathrm{XN}$ of [10] due to the $\mathrm{B} / \mathrm{R}$ module. TCLK-Q of DNVFF2 is lower by $11 \%$ w.r.t [10] due to lower $\mathrm{B} / \mathrm{R}$ module capacitance (Fig. 10(e)). Compared to volatile FF, $T_{C L K-Q}$ of DNVFF-2 is similar but that of DNVFF-1 is lower. This is because, transition at XN and $Q$ of DNVFF-1 occurs before PFEG switching in M6. Before $P$ switching, D-FEFETs exhibit lower capacitance (slope of $P$ - $V$ ) than standard FETs, which reduces the delay. $E_{O P}$ of DNVFF-1 includes $P$ switching energy of D-FEFETs, resulting in a $35 \% E_{O P}$ increase (for kinetic coefficient $\rho=2.5 \mathrm{ohm}-\mathrm{cm}$ ) w.r.t [10]. The EOP overhead can be mitigated by employing FE with lower $\rho$ (Fig. 10(f)). Recent experiments [14] show promising trends in this regard. For $\rho$ $=0.25 \mathrm{ohm}-\mathrm{cm}, E_{O P}$ penalty reduces to $14 \%$. DNVFF-2 overcomes the $E_{O P}$ overheads of DNVFF-1 with no $P$ switching during normal operation, achieving $E_{O P}$ similar to [10] and volatile FF (Fig. 10(f)).

\section{SYSTEM-LEVEL EVALUATION}

\subsection{Simulation Methodology}

To evaluate the system-level energy benefits of the proposed DNVFFs in the context of an intermittently-powered system, we use a modified version of the simulation framework presented in $[\underline{4}, \underline{19}]$. Our system-level analyses are based on the TI MSP430FR5739 MCU that runs at $24 \mathrm{MHz}$ [21], and uses a unified FEFET based 8KB 4T NVM [22]. NVFFs are used for designing the core registers (Fig. 11(a)). The system is powered using an energyharvesting source that charges a supply capacitor, $\mathrm{C}_{\mathrm{s}}(10 \mathrm{nF}$ or $100 \mathrm{nF}$ ). Note that, circuit results for the NVFFs shown earlier were for scaled technology (FinFETs). However, for compatibility with the TI MSP430 MCU, we re-design the D-FEFETs at $130 \mathrm{~nm}$ node (planar). The circuit-level energy/delay metrics of $130 \mathrm{~nm}$ node (Fig. 11(b)) show trends similar to FinFETs explained in Section 6.

\subsection{Results and Observations}

Here, we compare application-level energy consumption for DNVFF-1, DNVFF-2, NVFF [1ㅇ] and a standard volatile FF (STD), by running various synthetic and real benchmarks.

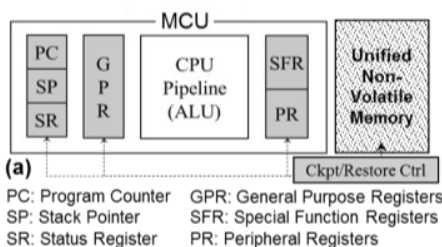

\begin{tabular}{|c|c|c|c|}
\hline \multicolumn{4}{|c|}{ Results for 130nm $\left(\mathrm{V}_{D D}=\mathbf{1 . 5 V}\right)$} \\
\hline & NVFF-1 & DNVFF-2 & NVFF [10] \\
\hline$E_{O P}$ & $12.7 \mathrm{fJ}$ & $9.3 \mathrm{fF}$ & $9.86 \mathrm{fJ}$ \\
\hline$T_{B}$ & $\sim 0 \mathrm{ps}$ & $298 \mathrm{ps}$ & $395 \mathrm{ps}$ \\
\hline$T_{R}$ & $135 \mathrm{ps}$ & $105 \mathrm{ps}$ & $103 \mathrm{ps}$ \\
\hline$E_{B}$ & $0.21 \mathrm{fJ}$ & $19.5 \mathrm{fJ}$ & $24.35 \mathrm{fJ}$ \\
\hline$E_{R}$ & $33.54 \mathrm{fJ}$ & $21.11 \mathrm{fJ}$ & $31.18 \mathrm{fJ}$ \\
\hline$L P$ & $161.4 \mathrm{pW}$ & $124.3 \mathrm{pW}$ & $149.1 \mathrm{pW}$ \\
\hline
\end{tabular}

Figure 11. (a) MCU Core Registers mapped to NVFF (b) energy/delay metrics of NVFFs at 130nm. LP: Leakage Power 

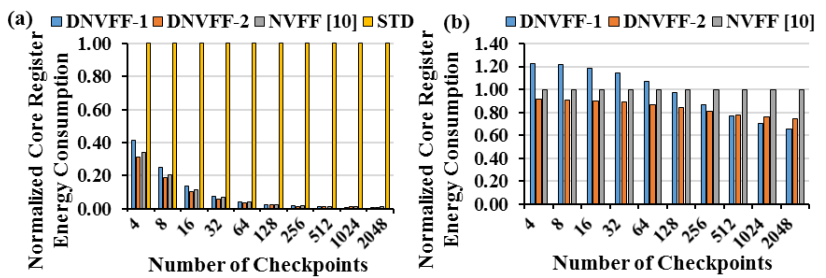

Figure 12. Normalized core register energy consumption of different NVFFs for synthetic benchmarks. The energies are normalized with (a) STD and (b) NVFF [ $\underline{10}]$ energies.

(i) Synthetic Benchmarks: Since, the energy benefits of using DNVFF-1, DNVFF-2 and NVFF [10] depend significantly on the number of system checkpoints (assumed to be hardware triggered) during application execution, we construct a synthetic benchmark with $25 \%$ of all instructions to be register reads, $25 \%$ to be register writes, and $50 \%$ to be memory bound instructions. In addition, we also vary the number of checkpoints (\#Ckpts) while keeping the checkpoint size (256 bytes) and total number of instructions (100K) constant (results in Fig. 12). Note that, in Fig. 12(a) and (b), the energies are normalized w.r.t the energy consumed by STD and NVFF [10], respectively. In Fig. 12(a), we observe that as \#Ckpts increase, the energy savings with NVFFs increase rapidly compared to the STD. This is because, the cost of check-pointing the core registers to the unified NVM (includes writing into NVM and serial transfer of checkpoint data) is orders of magnitude higher than the energy required to locally checkpoint in NVFFs. As a result, DNVFF-1, DNVFF-2 and NVFF [10] outperform STD FF (59\%-99\% better in energy consumption). From Fig. 12(b), we observe that for a small \#Ckpts, DNVFF-1 performs worse than either DNVFF-2 or NVFF [10] due to its relatively larger $E_{O P}$. However, as \#Ckpts increase, the overall energy consumption of DNVFF-1 starts to decrease w.r.t DNVFF2 and NVFF [10], because check-pointing energy of DNVFF-1 is substantially lower than that of DNVFF-2 and NVFF [10]. W.r.t NVFF [10], DNVFF-1 energy savings range from $23 \%$ worse (\#Ckpts=4) to $34 \%$ better (\#Ckpts=2048). On the other hand, the energy savings for DNVFF-2 are always better than NVFF [10] by 9\%-25\%. At lower \#Ckpts (<512), DNVFF-2 performs better than DNVFF-1 due to the former's lower EOP. However, at high \#Ckpts (>512), DNVFF-1 begins to outperform DNVFF-2.

(ii) Real Benchmarks: For real application benchmarks [18], we observe that DNVFF-1, DNVFF-2 and NVFF [10] showcase $99 \%$ and $98 \%$ energy improvements compared to STD on average for $\mathrm{C}_{\mathrm{s}}=10 \mathrm{nF}$ and $100 \mathrm{nF}$ respectively, which is consistent with our observation earlier. Fig. 13 shows the core register energy savings (normalized to NVFF [10]) achieved by DNVFF-1 and DNVFF-2 using real benchmarks. Fig. 13(a) demonstrates energy savings for DNVFF- 1 and DNVFF-2 of $33 \%$ and $25 \%$ on average respectively, w.r.t NVFF [10] for $\mathrm{C}_{\mathrm{s}}=10 \mathrm{nF}$. Fig. 13 (b) shows that DNVFF-1 and DNVFF-2 obtain $5 \%$ and $16 \%$ average energy savings respectively,

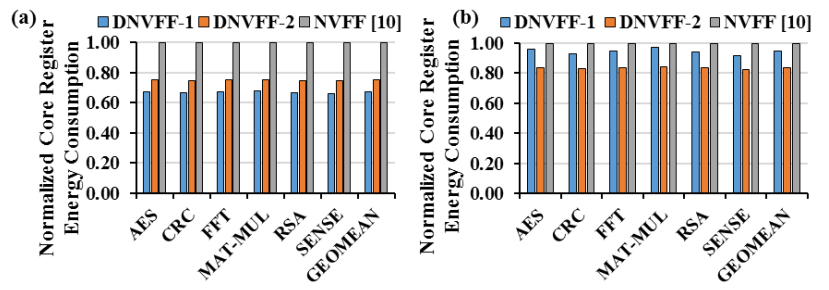

Figure 13. Normalized core register energy of different NVFFs for real benchmarks using (a) $\mathrm{C}_{\mathrm{s}}=10 \mathrm{nF}$ and (b) $\mathrm{C}_{\mathrm{s}}=100 \mathrm{nF}$ w.r.t NVFF [10] for $\mathrm{C}_{\mathrm{s}}=100 \mathrm{nF}$ (the savings are lower because, a larger capacitor leads to fewer checkpoints).

\section{CONCLUSION}

We propose D-FEFET, a specially designed FEFET, with a unique property of dynamic tuning between the volatile and nonvolatile modes of operations. Based on their intriguing properties, we propose two variants of NVFF designs. DNVFF-1 shows a complete automatic backup feature without the need of any external module, leading to lower backup/restore energy compared to NVFF in [10]. DNVFF-2 mitigates the operational energy costs of DNVFF-1 with improved backup energy/delay metrics compared to NVFF in [10]. Finally, we analyzed the system level implications of the DNVFFs showing a large promise for intermittently-powered systems.

\section{ACKNOWLEDGEMENT}

This research was supported in part by DARPA (\#D16AP00109), in part by NSF (\#1160483) and in part by the SRC (\#2016-SD-2723).

\section{REFERENCES}

[1]H. Jayakumar et al., "Powering the Internet of Things" IEEE/ACM Int. Symp. On Low Pow. Elec. Desgn. (ISLPED), pp. 375-380, 2014.

[2] C. Lu et al., "Analysis and design of ultra low power thermoelectric energy harvesting systems” in IEEE/ACM ISLPED, pp. 183-188, 2010.

[3] Y. Liu et al., "Ambient energy harvesting nonvolatile processors: From circuit to system" in Desgn. Auto. Conf. (DAC), pp. 1-6, 2015

[4] H. Jayakumar et al., "QuickRecall: A HW/SW Approach for Computing Across Power Cycles in Transiently Powered Computers". ACM f. Emerg. Technol. Comput. Syst., pp. 330-335, 2015.

[5]C. M. Jung et al., "Zerosleep-leakage flip-flop circuit with conditionalstoring memristor retention latch,” Trans. On Nanotech, pp. 360-366, 2012. [6] N. Sakimura et al., "Nonvolatile Magnetic Flip-Flop for Standby-PowerFree SoCs”, IEEE Journal of Solid-State Circuits, 44(8), pp. 355-358, 2009.

[7] I. Kazi et al., "Energy/Reliability Trade-Offs in Low-Voltage ReRAMBased Non-Volatile Flip-Flop Design” IEEE Trans. On Cir. and sys. I: Reg. Papers (TCAS), 61(11), pp. 3155-3164, 2014.

[8] H. Kimura et al., "A 2.4 pJ ferroelectric-based non-volatile flip-flop with 10-year data retention capability," in Proc. IEEE Asian Solid-State Circuits Conf. (A-SSCC), pp. 21-24, 2014.

[9] T. Böscke et al., "Ferroelectricity in Hafnium Oxide: CMOS Compatible Ferroelectric Field Effect Transistors," IEEE IEDM, pp. 24.5.1-24.5.4, 2011.

[10] X. Li et al., "Enabling energy-efficient nonvolatile computing with negative capacitance FET," Trans. on Elec. Dev. (TED), pp. 3452-3458 2017. [11] D. Wang et al., "Ferroelectric transistor based non-volatile flip-flop," in ISLPED, pp. 10-15, 2016.

[12] A. Aziz et al., "Physics-Based Circuit-Compatible SPICE Model for Ferroelectric Transistors", in IEEE Elec. Dev. Letts., 37(6), pp. 805-808, 2016. [13] M. Kobayashi et al., "Experimental study on polarization-limited operation speed of negative capacitance FET with ferroelectric HfO2", IEDM, pp. 12.3.1-12.3.4, Dec 2016.

[14] V. Boddhu et al., "Molecular dynamics study of ferroelectric domain nucleation and domain switching dynamics" Scientific Reports, April 2017. [15] A. I. Khan et al., "Ferroelectric negative capacitance MOSFET: Capacitance tuning \& antiferroelectric operation”, IEDM, 2011.

[16]https://newsroom.intel.com/newsroom/wp-

content/uploads/sites/11/2017/03/Kaizad-Mistry-2017-Manufacturing.pdf [17] S. Gupta et al., "Device-Circuit Analysis of Ferroelectric FETs for Low Power Logic", IEEE Trans. on Elec. Dev. (TED), 64(8), pp. 3092-3100, 2017 [18] S. K. Thirumala et al., "Gate Leakage in Non-Volatile Ferroelectric Transistors: Device-Circuit Implications” IEEE Dev. Res. Cof. (DRC), 2018. [19] A. Raha et al., "Designing energy-efficient intermittently powered systems using spin-hall-effect-based nonvolatile sram”. IEEE Trans. on VLSI Sys. (TVLSI), 26(2), pp. 294-307, 2018.

[20] https://www.mosis.com/files/scmos/scmos.pdf

[21] Texas Instruments. "MSP430FR573x Mixed-Signal-Microcontroller" [22] S. George et al., "Symmetric 2-D-Memory Access to Multidimensional Data” IEEE Trans. on VLSI Systems (TVLSI), 26(6), pp. 1-11, 2018. 